(Supporting Information) Effect of finite number of nanoblocks in metasurface lens design from Bloch-mode perspective and its experimental verification

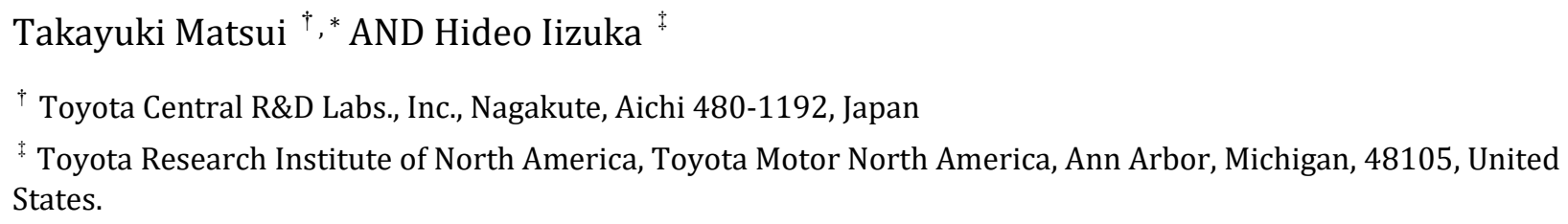

E-mail: t-matsui@mosk.tytlabs.co.jp 


\title{
[Supporting Information]: Effect of finite number of nanoblocks in metasurface lens design from Bloch-mode perspective and its experi- mental verification
}

\author{
Takayuki Matsui ${ }^{\dagger}, *$ AND Hideo Iizuka ${ }^{\ddagger}$ \\ $\dagger$ Toyota Central R\&D Labs., Inc., Nagakute, Aichi 480-1192, Japan \\ Toyota Research Institute of North America, Toyota Motor North America, Ann Arbor, Michigan, 48105, United \\ States.
}

KEYWORDS. metasurfaces, diffractive optics, wavefront control, flat optics, all-dielectric nanophotonics.

\section{SI1: Scattering from single nano-block}

Extinction for a single isolated nano-block was numerically calculated using a commercial finite element software (COMSOL with RF module). The nano-block was located on a fused silica substrate whose dimensions were $a r$, $a r$, and $h$ in width, depth, and height. $r$ is a fill-factor in the main text. The pitch of the unit cell and height of the block were set at $a=800 \mathrm{~nm}$ and $h=1100 \mathrm{~nm}$, respectively. The nano-block was irradiated from the substrate side with the normal incidence of $x$-polarized light. The background electric field was set based on analytical calculation as, $\mathrm{E}_{\mathrm{x}, \mathrm{in}}+\mathrm{E}_{\mathrm{x}, \mathrm{ref}}=$ $\exp \left(\mathrm{ik}_{1 \mathrm{z}} \mathrm{z}\right)+\mathrm{r}_{\mathrm{p}} \exp \left(-\mathrm{ik}_{1 \mathrm{z}}\right), \mathrm{E}_{\mathrm{x} \text {, tra }}=\mathrm{t}_{\mathrm{p}} \exp \left(\mathrm{ik}_{2 \mathrm{z}} \mathrm{z}\right)$, where $\mathrm{r}_{\mathrm{p}}$ and $t_{p}$ are the Fresnel coefficients at the substrate/air boundary. Forward and backward scatterings were calculated from the relative electromagnetic field with and without the nano-block, and the time-averaged Poynting vectors were normalized by the incident power in an area $a^{2}\left[\mathrm{~m}^{2}\right]$.

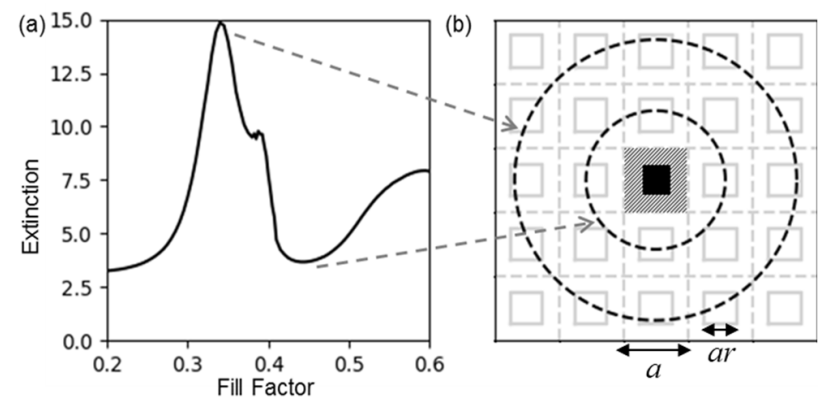

Fig. S1 (a) Extinction cross-section normalized by the unit cell area of $a^{2}$. (b) Schematic illustration of the cross-section from minimum (inner dotted ring) to maximum (outer dotted ring).

Figure S1 shows the extinction cross-section for the single nano-block. The extinction cross section ranged from 3 to 15 , which corresponds to a radius range of $1.0 a$ to $2.2 a$. Therefore, a silicon nano-block can interact with the first and second nearest neighboring nano-blocks when it is aligned in an array. Note that the time dependence in the software is exp $(j \omega t)$, and spatial dependence is expressed as exp $(-i k z)$. Care was taken when the results were compared with the periodic structure results calculated using a rigorous coupled wave analysis (RCWA) with exp $(-i \omega t)$.
SI2: Transmittance, Reflectance, and Transmission phase by periodic structure calculation

Meta-lenses were designed and fabricated based on the calculation by RCWA. Fig. S2 shows the transmittance (black solid line), reflectance (black dashed line), and transmission phase (gray dashed line) for the periodic structure $(a=800 \mathrm{~nm}, h=1100 \mathrm{~nm})$.

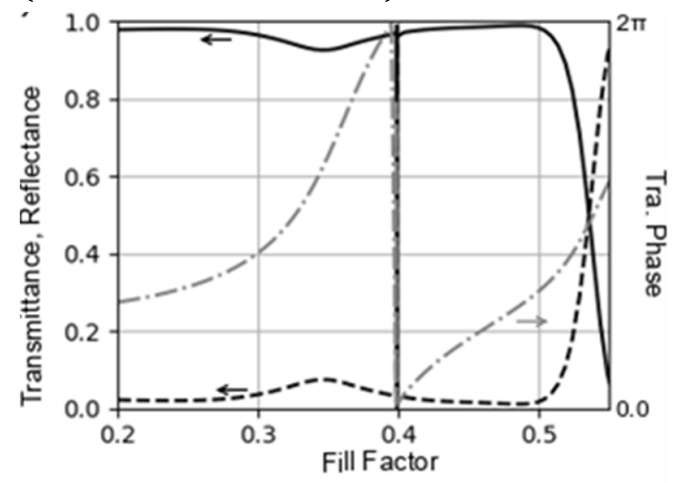

Fig. S2 Transmittance (solid line), reflectance (dashed line) , and transmission phase (dash-dotted line) obtained by the periodic structure calculation.

\section{SI3: Eigenvalue equation in RCWA}

Effective indices of modes were calculated based on Ref. [S1]. The eigenvalue equation is expressed as,

$$
k_{0}^{2} \boldsymbol{\Gamma}\left(\begin{array}{l}
\boldsymbol{E}_{y} \\
\boldsymbol{E}_{x}
\end{array}\right)=\left(j k_{z}\right)^{2}\left(\begin{array}{l}
\boldsymbol{E}_{y} \\
\boldsymbol{E}_{x}
\end{array}\right)
$$

where $\boldsymbol{\Gamma}$ is the block matrix

$$
\left[\begin{array}{cc}
\boldsymbol{K}_{x}^{2}-\left(\boldsymbol{I}-\boldsymbol{K}_{y} \boldsymbol{\epsilon}_{z}^{-1} \boldsymbol{K}_{\boldsymbol{y}}\right) \boldsymbol{\epsilon}_{y} & \boldsymbol{K}_{y}\left(\boldsymbol{\epsilon}_{z}^{-1} \boldsymbol{K}_{y} \boldsymbol{\epsilon}_{x}-\boldsymbol{K}_{x}\right) \\
\boldsymbol{K}_{x}\left(\boldsymbol{\epsilon}_{z}^{-1} \boldsymbol{K}_{y} \boldsymbol{\epsilon}_{y}-\boldsymbol{K}_{y}\right) & \boldsymbol{K}_{y}^{2}-\left(\boldsymbol{I}-\boldsymbol{K}_{x} \boldsymbol{\epsilon}_{z}^{-1} \boldsymbol{K}_{x}\right) \boldsymbol{\epsilon}_{x}
\end{array}\right],
$$

whose sub-matrix $\boldsymbol{K}_{\alpha}(\alpha=x, y)$ is a diagonal matrix, and $\boldsymbol{\epsilon}_{\beta}(\beta=x, y, z)$ is a Toeplitz matrix. $\boldsymbol{K}_{\alpha}$ is expressed as $\boldsymbol{K}_{\alpha}=$ $\operatorname{diag}\left(\bar{k}_{\alpha,-m,-m^{\prime}}, \bar{k}_{\alpha,-m,-m^{\prime}+1}, \bar{k}_{\alpha,-m,-m^{\prime}+2}, \ldots, \bar{k}_{\alpha,-m, m^{\prime}}\right.$, $\bar{k}_{\alpha,-m+1,-m^{\prime}}, \bar{k}_{\alpha,-m+1,-m^{\prime}+1}, \ldots, \bar{k}_{\alpha,-m+1, m^{\prime}}, \ldots, \bar{k}_{\alpha, m,-m^{\prime}}$, $\left.\bar{k}_{\alpha, m,-m^{\prime}+1}, \ldots, \bar{k}_{\alpha, m, m^{\prime}}\right)$, where $m\left(m^{\prime}\right)$ are truncation orders in the $\mathrm{x}(\mathrm{y})$-directions, $\bar{k}_{\alpha=x}=\left(k_{x, i n c}+\frac{2 \pi m}{a}\right) / k_{0}$, and $\bar{k}_{\alpha=y}=\left(k_{y, \text { inc }}+\frac{2 \pi m^{\prime}}{a}\right) / k_{0}$. Because the eigenvalue 
equation is expressed by the harmonic modes of the electric field [S2], $\boldsymbol{K}_{\mathrm{x}}$ and $\boldsymbol{K}_{\mathrm{y}}$ are different, and $\boldsymbol{\epsilon}_{x}$ and $\boldsymbol{\epsilon}_{\boldsymbol{y}}$ are different when we apply a fast factorization rule correctly[S3]; thus, the operator (Eq. S4) is not a Hermitian.

\section{SI4: Effective index by 2D Bloch analysis}

Table S1 shows the effective index $\left(n_{\text {eff }}=k^{g} / k_{0}\right)$ for modal analysis. The effective index $\left(n_{e f f}\right)$ and the electric field of modes $\left(\mathrm{E}^{\mathrm{g}}\right)$ were extracted using custom-built RCWA. Since eigenvalues and eigenvectors are independently determined with incident polarization, calculated eigenvectors are not always symmetric about the Cartesian coordinate of the $x$ - $(y$-)axis. Therefore, as mentioned in the main text, there are several modes that have the same eigenvalue. Modes with the same eigenvalue were merged to show fields and calculate contributions. Table S1 shows the effective indices and their contribution ratios with different fill-factors. Real valued $n_{\text {eff }}$ modes are shaded blue in the table, and modes that have complex-valued $n_{\text {eff }}$ are shaded red. As seen in the table, for fill-factors of 0.40, 0.45, and 0.50 , two propagating modes (real-valued $n_{\text {eff }}$ ) in addition to a hybrid Bloch mode are excited. Fig. S3 shows contour plots of the modes.
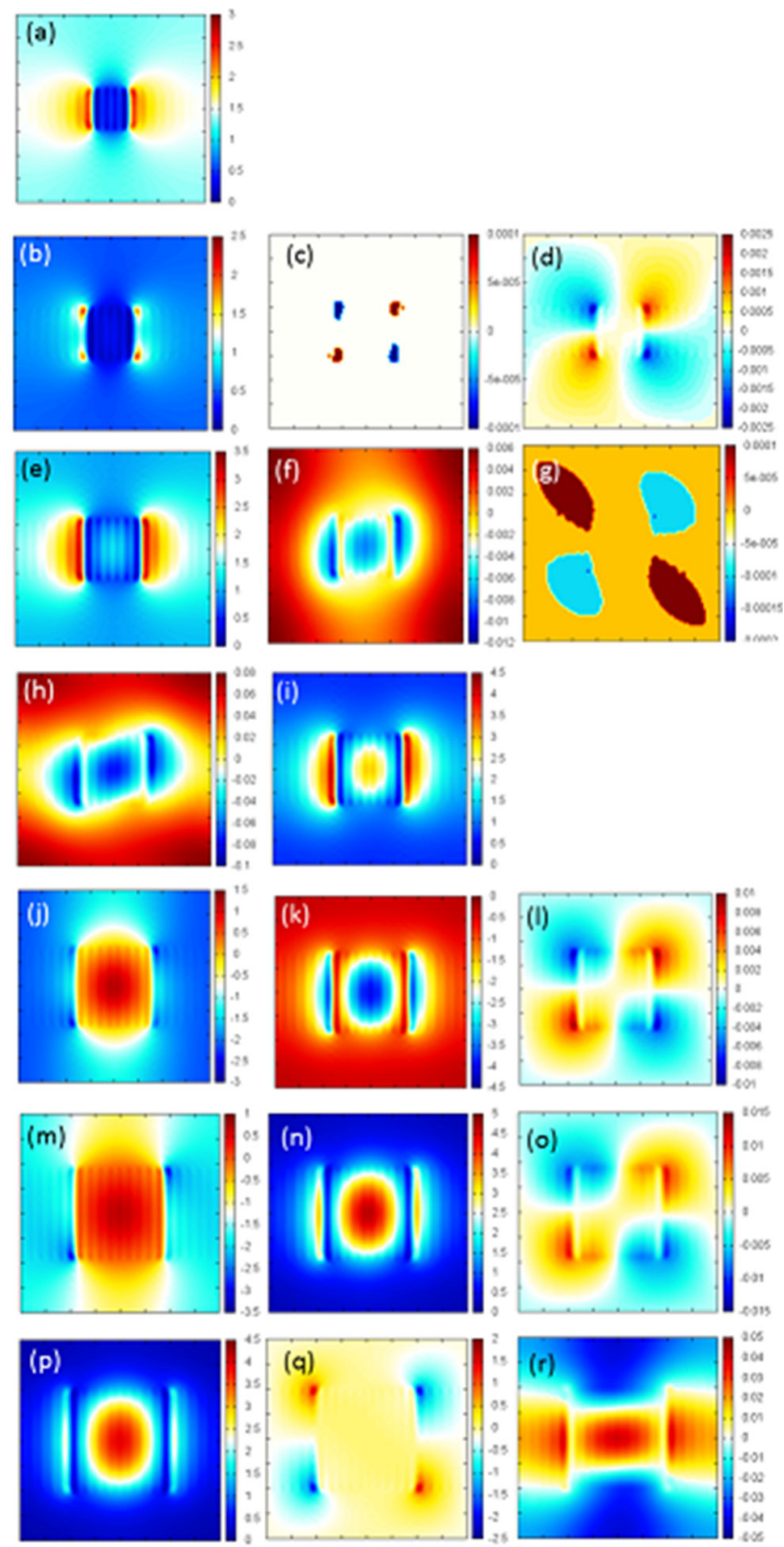

Fig. S3 Field distributions for main modes for various fill factors. (a) $\mathrm{r}=0.2$ and $n_{\text {eff }}=1.055$, (b) $\mathrm{r}=0.25$ and $n_{\text {eff }}=1.113$, (c) $\mathrm{r}=$ 0.25 and $n_{\text {eff }}=0.294+2.368 \mathrm{i}$, (d) $\mathrm{r}=0.25$ and $n_{\text {eff }}=0.216+1.642 \mathrm{i}$, (e) $\mathrm{r}=0.3$ and $n_{\text {eff }}=1.256$, (f) $\mathrm{r}=0.3$ and $n_{\text {eff }}=0.496+1.378 \mathrm{i},(\mathrm{g}) \mathrm{r}=0.3$ and $n_{\text {eff }}=0.064+2.134 \mathrm{i},(\mathrm{h}) \mathrm{r}=$ 0.35 and $n_{\text {eff }}=0.213+0.848 \mathrm{i}$, (i) $\mathrm{r}=0.35$ and $n_{\text {eff }}=1.646$, (j) $\mathrm{r}=$ 0.4 and $n_{\text {eff }}=0.648$, (k) $\mathrm{r}=0.4$ and $n_{\text {eff }}=2.1$, (l) $\mathrm{r}=0.4$ and $n_{\text {eff }}=1.111 \mathrm{i},(\mathrm{m}) \mathrm{r}=0.45$ and $n_{\text {eff }}=0.814$, (n) $\mathrm{r}=0.45$ and $n_{\text {eff }}=2.411$, (o) $\mathrm{r}=0.45$ and $n_{\text {eff }}=1.071 \mathrm{i},(\mathrm{p}) \mathrm{r}=0.5$ and $n_{\text {eff }}=2.624$, (q) $\mathrm{r}=0.5$ and $n_{\text {eff }}=0.891$, (r) $\mathrm{r}=0.5$ and $n_{\text {eff }}=0.912 \mathrm{i}$. Truncation orders in RCWA calculation are $m=m^{\prime}=20$. 
Table S1 Effective indices of main modes and their contribution ratios. Main modes are sorted by contribution ratios.

\begin{tabular}{l|l|l|l}
$r \backslash$ mode\# & $1^{\text {rl }}$ mode & $2^{\text {rd }}$ mode & $3^{\text {rd }}$ mode \\
\hline 0.20 & 1.055 & & \\
& $(98.6 \%)$ & & \\
\hline 0.25 & 1.113 & $0.294+2.368 \mathrm{i}$ & $0.216+1.642 \mathrm{i}$ \\
& $(95.7 \%)$ & $(1.6 \%)$ & $(1.4 \%)$ \\
\hline 0.30 & 1.256 & $0.496+1.378 \mathrm{i}$ & $0.064+2.134 \mathrm{i}$ \\
& $(84.7 \%)$ & $(11.3 \%)$ & $(3.7 \%)$ \\
\hline 0.35 & $0.213+0.848 \mathrm{i}$ & 1.646 & \\
& $(70.7 \%)$ & $(28.9 \%)$ & \\
\hline 0.40 & 0.648 & 2.10 & $1.111 \mathrm{i}$ \\
& $(53.5 \%)$ & $(39.5 \%)$ & $(6.4 \%)$ \\
\hline 0.45 & 0.814 & 2.411 & $1.071 \mathrm{i}$ \\
& $(65.6 \%)$ & $(27.9 \%)$ & $(5.9 \%)$ \\
\hline 0.50 & 2.624 & 0.891 & $0.912 \mathrm{i}$ \\
& $(47.6 \%)$ & $(41.5 \%)$ & $(10.1 \%)$
\end{tabular}

Fig. S3 represents the modal field distributions listed in Table S1. Modes with contributions larger than $1.0 \%$ are depicted as sign $(\arg (\operatorname{Ex}(\mathrm{x}, \mathrm{y})))|\operatorname{Ex}(\mathrm{x}, \mathrm{y})|$. The width and height of images are $a=800 \mathrm{~nm}$. Some quadrupole modes are observed in Fig. S3d, l, o, and q. The modes in Fig. S3 c and g seem to contribute to RCWA convergence, and have less physical meaning. The effective indices table (Table S1) and field distributions (Fig. S3) indicate that the operation mechanism is attributed to EMT $(r=0.25)$, scattering $(r=$ $0.3,0.35)$, and the hybrid-Bloch mode $(r=0.40,0.45,0.50)$. A fill factor of $r=0.25$ is somewhere between EMT and scattering.

RCWA is a rigorous electromagnetic calculation method in which relative permittivity distributions are expressed by Fourier expansion within limited truncation orders. Given the truncation order expressed by $m$ and $m$ 'for the $x^{-}$ and $y$-directions, the number of total modes is $2(2 m+1)\left(2 m^{\prime}+1\right)$; e.g. 3362 modes when $\left(m, m^{\prime}\right)=(20,20)$. A few modes have physical meaning, but other modes contribute to convergence.

Our RCWA code is implemented based on Ref. [S1, S4, S5], and we confirmed that our code reproduces the results of reported papers, for example, Refs. [S6-S14].

\section{SI5: Forward scattering ratio calculations}

We compare the forward scattering of isolated nanoblocks with that of the periodic structure. Because a periodic structure assumes an infinite array of nano-blocks, the diffraction wave of each order has no angular variation arising from diffraction through a limited aperture. Therefore, we compare the forward ratio from far-fields for the direction $(\theta=0, \phi=0)$ as,

$$
F R=\frac{n_{\text {Air }}\left|E_{f a r}(\theta=0, \phi=0)\right|^{2}}{n_{\text {Air }}\left|E_{f a r}(\theta=0, \phi=0)\right|^{2}+n_{S u b}\left|E_{f a r}(\theta=\pi, \phi=0)\right|^{2}},
$$

and this is plotted in Fig. 3a. The plots in Fig. S4 are based on the scattering ratio calculated as $F R=F S /(F S+B S)$ where $F S$ and $B S$ are calculated from the time-averaged Poynting vector of the upper hemisphere and the lower hemisphere, respectively. While the forward ratio for the upper hemisphere is independent of the number of nanoblocks (as in Fig. S4), the directivity $(\theta=0, \phi=0)$ converges to the periodic structure calculation (gray solid line, Fig. 3a) as the number of nano-blocks increases.

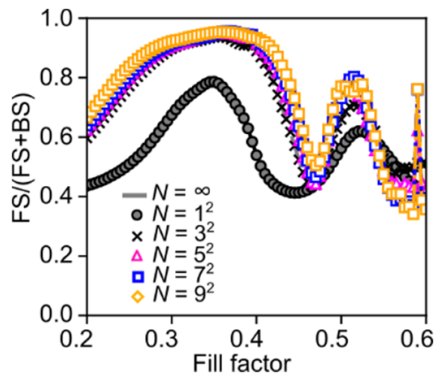

Fig. S4 Forward ratios calculated from the time-averaged Poynting vector for the upper hemisphere divided by the whole sphere.

\section{SI6: Field distribution comparison between periodic} structure and PML terminated isolated structure.

Electric field distributions $\left(\operatorname{Re}\left(E_{x}\right)\right)$ with the same nanoblock sizes were compared between the periodic structure (Fig. S5 upper) and the PML terminated isolated structure (Fig. S5 lower). The number of nano-blocks for the isolated condition was $N=5^{2}$.

As pointed out in the main text, the FR through the isolated structure converges to the transmittance of the periodic structure. An FR dip is observed around $r=0.45$. Considering both field distributions reasonably agreed, the electromagnetic response from both calculations would become identical as the number of isolated nano-blocks increases, if computational resources permit.

The periodic structure was calculated by both RCWA and FEM (commercial software, COMSOL), and identical transmittance and reflectance were confirmed.

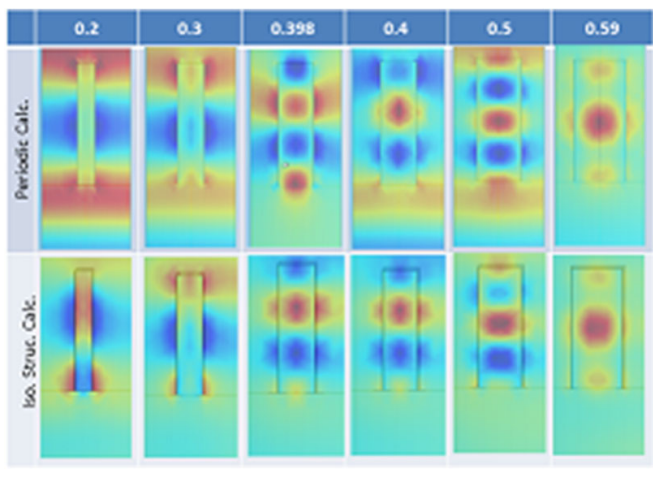

Fig. S5 Electric field distribution comparison between (a) the periodic boundary condition (upper) and (b) the isolated condition (lower). The numbers in the upper row depict the fillfactor. The width of the rendered region corresponds to the pitch of the unit cell.

SI7: Calculated contour plots and their cut-lines 
Transmittance contours corresponding to Figs. 5g-i are rendered in Fig. S6. Contours are based on the calculations of different numbers of isolated nano-blocks, $\mathrm{N}=3^{2}, 5^{2}, 7^{2}$, and $9^{2}$, respectively, from the top row. The bottom row shows cut-line plots along the upper side of each figure, which correspond to Figs. $5 \mathrm{j}-1$ in the manuscript. Color relations are the same: $N=3^{2}$ (black), $5^{2}$ (magenta), $7^{2}$ (blue), $9^{2}$ (orange). Considering that (i) periodic calculations cannot reproduce the experiment, and that (ii) the orange line $\left(\mathrm{N}=9^{2}\right)$ shows the closest relevance with the experiments (gray circle plots), short-range periodicity is the dominant operating mechanism for meta-lenses.

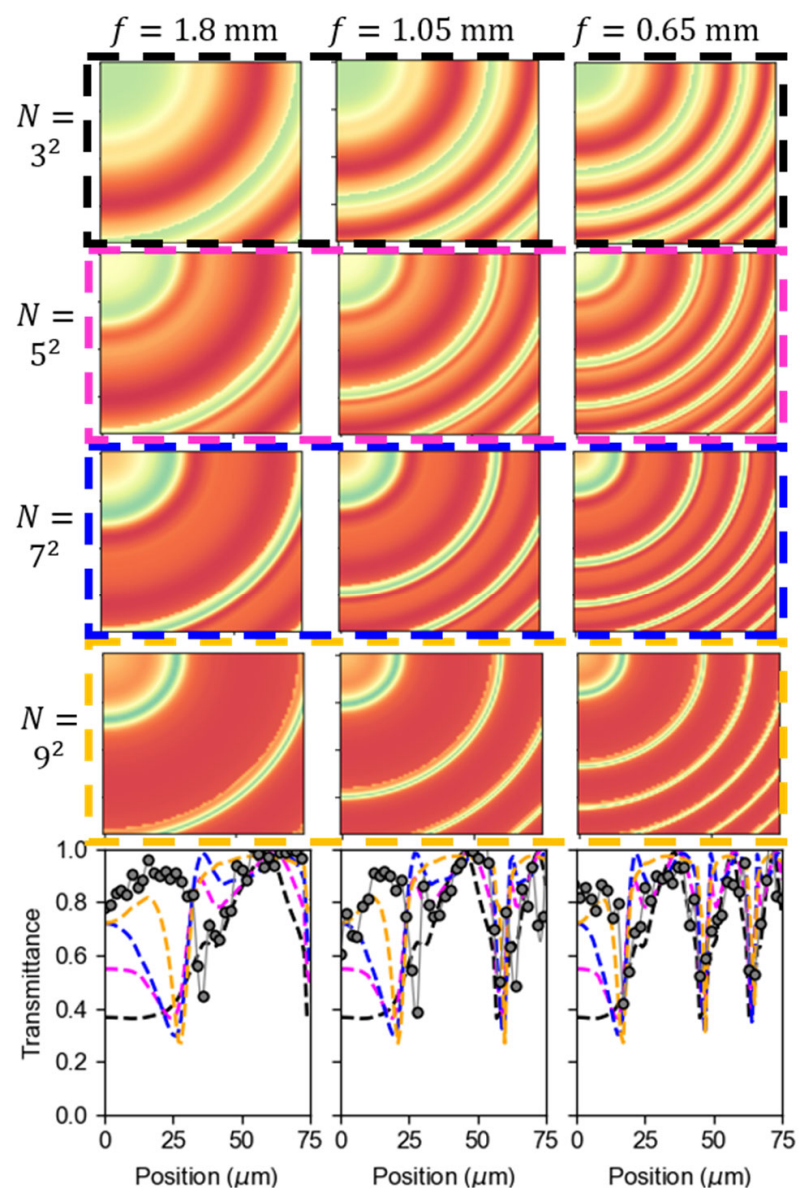

Fig. S6 Transmittance contours based on the calculations for isolated nano-blocks. The bottom row plots are cut-lines for the corresponding upper side of the contours. (black dashed line: $N=3^{2}$, magenta dashed line: $N=5^{2}$, blue dashed line: $N=7^{2}$, orange dashed line: $N=9^{2}$, gray symbols: experiment.)

\section{SI8: Focused profile at foci}

Focal intensity profiles $\left(I=|E|^{2}\right)$ through the meta-lens were calculated based on the Fresnel diffraction integral using a paraxial approximation:

$$
\begin{aligned}
& E(x, y, z)=e^{i k z} / i \lambda z \int_{-\infty}^{\infty} d x_{0} \int_{-\infty}^{\infty} d y_{0} A\left(x_{0}, y_{0}\right) e^{i k / 2 z}[(x- \\
& \left.\left.\left.x_{0}\right)^{2}+\left(y-y_{0}\right)\right)^{2}\right],(\mathrm{S} 2)
\end{aligned}
$$

,where $A\left(x_{0}, y_{0}\right)$ is the electric field distribution in the plane of the meta-lens acquired from the numerical calculation from Fig. 3 (the isolated structure, in the main text) and Fig. S2 (the periodic structure).
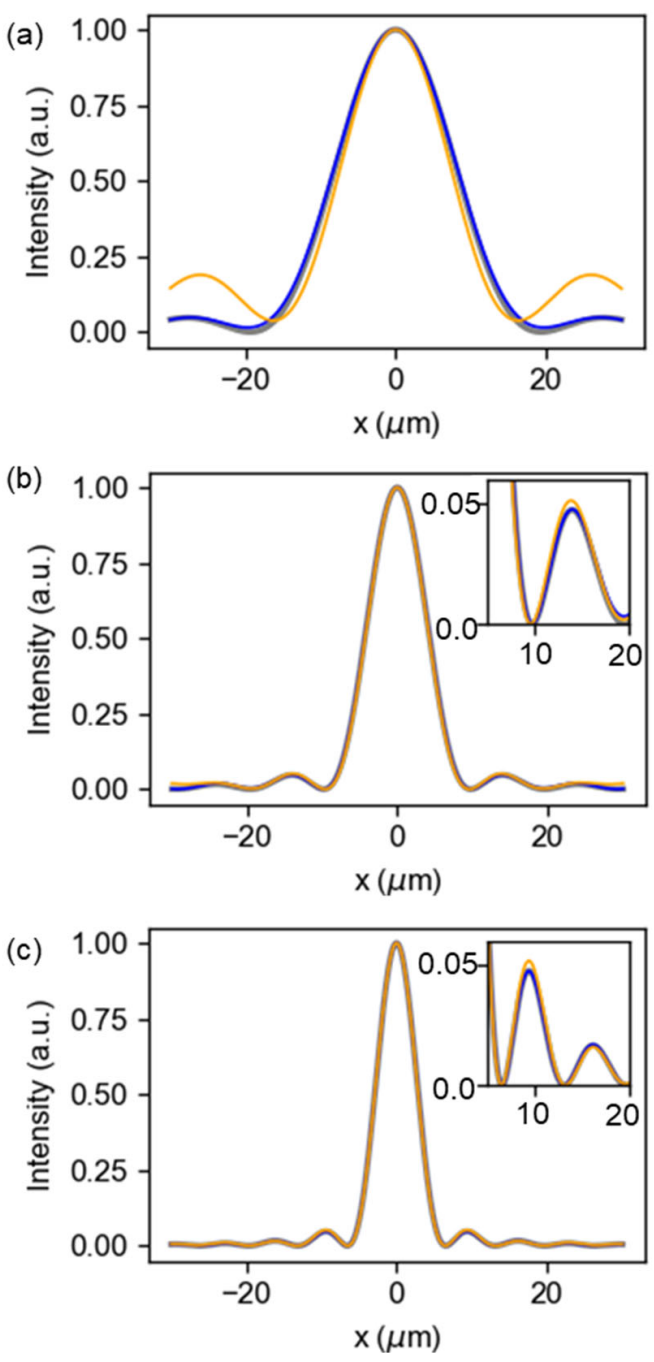

Fig. S7 Calculated focal intensity profiles through meta-lenses. Thelens aperture width is set at (a) $50 \mu \mathrm{m}$, (b) $100 \mu \mathrm{m}$, and (c) $150 \mu \mathrm{m}$ with a fixed focal length of $650 \mu \mathrm{m}$.

Cut-line intensity profiles are shown in Fig. S7. The phase and amplitude distributions for the lens were set as follows. (i) The target phases in the plane were set based on $\phi\left(x_{0}, y_{0}\right)=2 \pi / \lambda\left(\sqrt{f^{2}+x^{2}+y^{2}}-f\right)$. (ii) The corresponding fill-factors at the lens position $\left(x_{0}, y_{0}\right)$ were determined from periodic calculation results (Fig. S2). (iii) The phase and amplitude distributions were set according to this fillfactor from Fig. S2 (periodic structure-based) and Fig. 3 (isolated structure-based). The solid gray line in Fig. S7 corresponds to the profile through an ideal distribution of $A\left(x_{0}, y_{0}\right)=e^{i \phi\left(x_{0}, y_{0}\right)}$. The blue line represents the profile based on the periodic structure calculation (Fig. S2), and the orange line represents that based on the isolated structure (Fig. 3 in the main text). Because the spatial resolution is inversely proportional to the spread of the incident 
wavevector, focal intensity profiles become narrower as the aperture size increases. As pointed out in the main text, the intensity profile of the outer bright ring shows a discrepancy from the ideal distribution due to the incorrect distribution of the lens (orange line), which becomes apparent with a narrower aperture.

\section{Corresponding Author}

*t-matsui@mosk.tytlabs.co.jp

\section{Author Contributions}

The manuscript was written through contributions of all authors.
S2. J. D. Joannopoulos et al., "Photonic Crystals -Molding the Flow of Light- $2^{\text {nd }}$ Edition", Princeton University Press (2008).

S3. L. Li J. Opt. A Pure Appl. Opt. 5, 345-355 (2003).

S4. M. G. Moharam, et al., J. Opt. Soc. Am. A 12(5), 1068-1076 (1995).

S5. T. Weiss, et al., J. Opt, A-Pure Appl. Op. 11(11), 114019 (2009).

S6. (Fig.2a) I. Staude, et al., ACS Nano 7, 7824-7832 (2013).

S7. (Fig.2a) P. Bouchon, et al., Appl. Phys. Lett. 98, 191109 (2011).

S8. (Fig.3c) P. Moitra, et al., Appl. Phys. Lett. 104, 171102 (2014).

S9. (Fig.2a) P. Spinelli, et al., Nature Communications 3:692 (2012). S10. (Fig.2b) M. Shokooh-Saremi et al., Opt. Lett. 32, 894-896 (2007).

S11. (Fig.1b) P. Bouchon, et al., Opt. Lett. 37, 1038-1040 (2012).

S12. (Fig.4) M. Shokooh-Saremi, et al., Opt. Lett. 39, 6958-6961 (2014)

S13. (Fig.7) S. Fan, et al., Phys. Rev. B 65, 235112 (2002).

S14. [Fig.2b] G. Dolling et al., Opt. Lett. 31 1800-1802 (2006).

S15. L. Novotny and B. Hecht, Principles of Nano-Optics. Cambridge University Press, 2012.

S16. H. A. Haus, "Waves and Fields in Optoelectronics," PrenticeHail, Inc. (1984).

\section{REFERENCES}

S1. H. Kim, et al., "Fourier modal method and its applications in computational nanophotonics." CRC Press, 2012. 